\title{
Excited-State and Reactive Clusters: Synthesis, Structure, Reactivities, and Properties
}

\author{
Boon K. Teo
}

Published online: 29 March 2013

(C) Springer Science+Business Media New York 2013

This is a special issue on "Excited-State and Reactive Clusters: Synthesis, Structure, Reactivities, and Properties." It contains two reviews and 13 original papers. The subject matters range from experimental to theoretical studies of excited-state or reactive clusters as they relate to, or are involved in, interesting chemical (such as catalysis) and physical properties (e.g., luminescence). The materials involved range from zero-dimensional (0-D) quantum dots to one-dimensional (1-D) nanowires or nanotubes to two-dimensional (2-D) hydrogen bonded systems to three-dimensional (3-D) polyhedral structures. New synthetic methods, novel structures, and unusual chemical bonding of clusters, especially those related to excited or reactive clusters, are also reported.

The subject matters are highlighted below:

In the invited review entitled "Excited State Relaxation and Stabilization of Hydrogen-Terminated Silicon Quantum Dots," Wu, Wang, Li, and Zhang provide a comprehensive review of recent advances in the theoretical investigation of the excited state properties of silicon quantum dots (QDs). The theoretical calculations revealed that the size-dependent excited-state relaxation due to surface passivation of silicon QDs by hydrogen can critically impact physical properties such as photoluminescence and hence their ultimate technological applications.

The invited review entitled "Excited Semiconductor Clusters: Magic Source," by Yang, Wang, and Zhang deals with the preparation and properties of CdS-like clusters as well as their applications. The formation of nanostructures, including 1-D solid and tubular fibers, 2-D dendritic morphology, and 3-D crystals are reviewed and their photoluminescence discussed. A specific example is provided in a related

\section{B. K. Teo $(\bowtie)$}

College of Chemistry and Chemical Engineering, Xiamen University, Xiamen,

Fujian 361005, China

e-mail: boonkteo@gmail.com; boonkteo@xmu.edu.cn

B. K. Teo

College of Chemistry and Molecular Engineering, Peking University, Beijing 100871, China 
paper entitled "Photoluminescence Stability of Colloidal CdTe Quantum Dots in Various Buffer Solutions" where P. Yang and coworkers evaluate the use of mercaptoacetic acid-capped CdTe quantum dots (QDs) as potential luminescent markers for biological analysis by monitoring their stability in electrophoresis buffers.

In the invited paper entitled "Modeling and Simulation of Bonding and Optical Characters of Ternary Nanocrystals," Wang, Xu, Shao, Wang, and Cui, provide a summary of their theoretical calculations on conformations of the ternary structures $\mathrm{ZnSe}(\mathrm{Te}), \mathrm{ZnS}(\mathrm{Te}), \mathrm{ZnS}(\mathrm{Se}), \mathrm{CdSe}(\mathrm{Te}), \mathrm{CdS}(\mathrm{Te})$ and $\mathrm{CdS}(\mathrm{Se})$ and their optical spectra as a function of doping.

In the invited paper entitled "Novel $\mathrm{pH}$ Induced Reversible Luminescent Lanthanide Hydrogels," Zheng, Pang, Zhou, Wang, Tan, and Zhong report the photoluminescence of two lanthanide $\operatorname{Ln}(\mathrm{III})$-containing hydrogels which can be switched "on-and-off' reversibly by a change in $\mathrm{pH}$.

Papers 6 to 9 deal with theoretical studies of electronically excited states of hydrogen bonded systems. In Paper 6, entitled “Time-Dependent Density Functional Theory Study on Hydrogen and Dihydrogen Bonding in Electronically Excited State of 2-Pyridone-Borane-Trimethylamine Cluster," C. Hao and coworkers explore the intermolecular hydrogen and dihydrogen bonding interactions in electronically excited states of a 2-pyridone (2PY)-borane-trimethylamine cluster using timedependent density functional theory (TDDFT). Paper 7, entitled "A Theoretical Study on Electronically Excited States of the Hydrogen-Bonded Clusters for Fluorenone and Fluorenone Derivatives in Methanol Solvent," is a report by W. Zhang and coworkers on TDDFT calculations of electronically excited states of hydrogen-bonded complexes of fluorenone (FN), FN derivatives, and methanol (as solvent). Paper 8, entitled "Effects of Hydrogen Bonding on the Transition Properties of Ethanol-Water Clusters: A TD-DFT Study," is a similar study by D. Yang and coworkers on the electronic transitions of hydrogen-bonded ethanol-water complexes. In Paper 9, entitled "Influence of Hydrogen Bonding and Polarity on the Spectral Properties of 4-Aminophthalimide Clusters Formed with Triethylamine and DimethylSulfoxide in Solution," D. Yang et al report a TDDFT study of the influences of hydrogen bonding and solvent polarity on the spectral properties of 4-aminophthalimide clusters formed with hydrogen accepting solvents triethylamine and dimethyl sulfoxide.

Finally, Papers 10 to 15 are reports on reactive or activated clusters or nanofibers exhibiting interesting catalytic or photocatalytic activities. Specifically, in Paper 10, entitled "Study on Photocatalytic Activity of $\mathrm{NaTaO}_{3}$ Powder Synthesized by Hydrothermal Method," W. Tan and coworkers prepared $\mathrm{NaTaO}_{3}$ powders by hydrothermal method and investigated their photocatalytic activities. In Paper 11, entitled "One-Dimensional $\mathrm{Bi}_{2} \mathrm{WO}_{6}$ Nanofibers Controllable Synthesis by Electrospinning and Enhanced Visible Photocatalytic Degradation Performances," 1-D $\mathrm{Bi}_{2} \mathrm{WO}_{6}$ nanofibers were synthesized by electrospinning and their photocatalytic activity investigated by S. Liu and coworkers. In Paper 12, entitled "Hydrothermal Synthesis and Characterization of Visible-Light-Driven Dumbbell-Like $\mathrm{BiVO}_{4}$ and $\mathrm{Ag} / \mathrm{BiVO}_{4}$ Photocatalysts," W. Que and coauthors synthesized the visible-lightdriven dumbbell-shaped $\mathrm{BiVO}_{4}$ and $\mathrm{Ag} / \mathrm{BiVO}_{4}$ photocatalysts via the hydrothermal method and found significant enhancement of the photocatalytic activity of $\mathrm{BiVO}_{4}$ 
as a result of the incorporation of $\mathrm{Ag}$ nanoparticles. In Paper 13, entitled "Synthesis and Structure of $\left[\mathrm{Co}_{2} \mathrm{Bi}_{2}\left(\alpha \text { or } \beta-\mathrm{B}-\mathrm{CoW}_{9} \mathrm{O}_{34}\right)_{2}\right]^{14-}$ Isomers and Their Catalytic Water Oxidation," the title clusters were synthesized by W. You and coworkers and shown to exhibit visible-light-induced catalytic activity involving the electrochemical oxidation of $\mathrm{H}_{2} \mathrm{O}$ to yield $\mathrm{O}_{2}$. Paper 14, entitled "Application of Solid-State Molybdenum Sulfide Clusters with an Octahedral Metal Framework to Catalysis: Ring-Opening of Tetrahydrofuran to Butyraldehyde," deals with the investigation on the use of the superconducting Chevrel phases to catalysis by S. Kamiguchi and coworkers. It was shown that, by treating the cluster $\mathrm{Cu}_{\mathrm{x}} \mathrm{Mo}_{6} \mathrm{~S}_{8}(\mathrm{x}=2.94)$ in a hydrogen stream above $300{ }^{\circ} \mathrm{C}$, the activated cluster exhibited catalytic activity for the ring opening of tetrahydrofuran to form butyraldehyde. Finally, in Paper 15, entitled "Evolution of $(\mathrm{GaAl})_{\mathrm{n}}$ Clusters and Chemisorptions of $\mathrm{H}_{2}$ on $(\mathrm{GaAl})_{\mathrm{n}}$ Clusters," L. Guo carried out theoretical calculations to investigate the growth behavior of $(\mathrm{GaAl})_{n}(\mathrm{n}=1-12)$ clusters and the chemisorption sites of hydrogen on these clusters. 